\title{
When does the associated graded Lie algebra of an arrangement group decompose?
}

\author{
Stefan Papadima* and Alexander I. Suciu ${ }^{\dagger}$
}

\begin{abstract}
Let $\mathcal{A}$ be a complex hyperplane arrangement, with fundamental group $G$ and holonomy Lie algebra $\mathfrak{H}$. Suppose $\mathfrak{H}_{3}$ is a free abelian group of minimum possible rank, given the values the Möbius function $\mu: \mathscr{L}_{2} \rightarrow \mathbb{Z}$ takes on the rank 2 flats of $\mathcal{A}$. Then the associated graded Lie algebra of $G$ decomposes (in degrees $\geq 2$ ) as a direct product of free Lie algebras. In particular, the ranks of the lower central series quotients of the group are given by $\phi_{r}(G)=\sum_{X \in \mathscr{L}_{2}} \phi_{r}\left(F_{\mu(X)}\right)$, for $r \geq 2$. We illustrate this new Lower Central Series formula with several families of examples.
\end{abstract}

Mathematics Subject Classification (2000). Primary 52C35; Secondary 20F14, 20F40.

Keywords. Hyperplane arrangement, lower central series, associated graded Lie algebra, holonomy Lie algebra, Chen Lie algebra.

\section{Introduction}

1.1. The purpose of this paper is to give an answer to the question posed in the title. Let $\mathcal{A}$ be an arrangement of finitely many hyperplanes through the origin of $\mathbb{C}^{\ell}$, and denote by $G(\mathcal{A})=\pi_{1}\left(\mathbb{C}^{\ell} \backslash \bigcup_{H \in \mathcal{A}} H\right)$ the fundamental group of its complement. In Section 2, we single out a class of arrangements, closely related to certain arrangements studied in [2], [15]. Roughly speaking, $\mathcal{A}$ is decomposable if a certain quadratic, graded Lie algebra $\mathfrak{H}(\mathcal{A})$, naturally defined in terms of the codimension 2 flats of $\mathcal{A}$, has minimal possible dimension in degree 3 , over any ground field.

Our main result (Theorem 2.4) implies the following: If $\mathcal{A}$ is decomposable, then the associated graded Lie algebra of $G(\mathcal{A})$ decomposes as a direct product of free Lie algebras (in degrees $r \geq 2$ ):

$$
\operatorname{gr}_{\geq 2}(G(\mathcal{A})) \cong \prod_{X \in \mathscr{L}_{2}(\mathcal{A})} \operatorname{gr}_{\geq 2}\left(F_{\mu(X)}\right)
$$

\footnotetext{
*Partially supported by CERES grant 152/2003 of the Romanian Ministry of Education and Research.

${ }^{\dagger}$ Partially supported by NSF grant DMS-0311142.
} 
Here:

- $\mathcal{L}(\mathcal{A})=\left\{X=\bigcap_{H \in \mathscr{B}} H \mid \mathscr{B} \subseteq \mathcal{A}\right\}$ is the intersection lattice, $\mathscr{L}_{2}(\mathcal{A})$ is the set of codimension 2 flats, and $\mu: \mathcal{L}(\mathcal{A}) \rightarrow \mathbb{Z}$ is the Möbius function.

- $\left\{\Gamma_{r} G\right\}_{r \geq 1}$ is the lower central series, given by $\Gamma_{1} G=G$ and $\Gamma_{r+1} G=\left(\Gamma_{r} G, G\right)$.

- $\operatorname{gr}(G)=\bigoplus_{r \geq 1} \Gamma_{r} G / \Gamma_{r+1} G$, with Lie bracket induced by the group commutator.

- $F_{n}$ is the free group of rank $n$, and $\operatorname{gr}\left(F_{n}\right)=\boldsymbol{L}_{n}$ is the free Lie algebra on $n$ generators.

Moreover, as we show in Proposition 3.3, the decomposability property of $\mathcal{A}$ is inherited by all sub-arrangements of $\mathcal{A}$.

1.2. The associated graded Lie algebra $\operatorname{gr}(G(\mathcal{A}))$ is not a priori determined by the intersection lattice, and, as such, it is not easy to handle. We turn instead to a more manageable, combinatorial approximation: The holonomy Lie algebra of the arrangement, $\mathfrak{H}(\mathcal{A})$, defined as the quotient of $\boldsymbol{L}(\mathcal{A})$, the free Lie algebra on variables $\left\{x_{H} \mid H \in \mathcal{A}\right\}$, modulo the ideal $J(\mathcal{A})$ generated by relations corresponding to rank 2 flats:

$$
\mathfrak{H}(\mathcal{A})=\boldsymbol{L}(\mathcal{A}) / \text { ideal }\left\{\left[x_{H}, \sum_{H^{\prime} \in \mathcal{A}: H^{\prime} \supset X} x_{H^{\prime}}\right] \mid X \in \mathscr{L}_{2}(\mathcal{A}) \text { and } X \subset H\right\} \text {. }
$$

As shown by Kohno [6] (based on foundational work by Sullivan [18] and Morgan $[10])$, the associated graded Lie algebra $\operatorname{gr}(G(\mathcal{A}))$ and the holonomy Lie algebra $\mathfrak{H}(\mathcal{A})$ are rationally isomorphic:

$$
\operatorname{gr}(G(\mathcal{A})) \otimes \mathbb{Q} \cong \mathfrak{H}(\mathcal{A}) \otimes \mathbb{Q} .
$$

At the integral level, there is a surjective Lie algebra map, $\Psi_{\mathcal{A}}: \mathfrak{H}(\mathcal{A}) \rightarrow \operatorname{gr}(G(\mathcal{A})$ ), such that $\Psi_{\mathcal{A}} \otimes \mathbb{Q}$ is an isomorphism, see [9]. In general, there exist arrangements for which $\Psi_{\mathcal{A}}$ is not injective. Nevertheless, for the class of decomposable arrangements we consider here, $\Psi_{\mathcal{A}}$ gives an isomorphism $\operatorname{gr}(G(\mathcal{A})) \cong \mathfrak{H}(\mathcal{A})$, see Theorem 2.4(2).

1.3. The lower central series ranks of a finitely-generated group $G$ are defined as $\phi_{r}(G)=\operatorname{rank~gr}_{r}(G)$. For a free group, the LCS ranks are given by Witt's formula: $\prod_{r=1}^{\infty}\left(1-t^{r}\right)^{\phi_{r}\left(F_{n}\right)}=1-n t$. For an arrangement group, the LCS ranks are determined by the intersection lattice, via (1.3) and (1.2). Clearly, $\phi_{1}(G(\mathcal{A}))=|\mathcal{A}|$. Hence, to determine the LCS ranks of $G(\mathcal{A})$, we only need to compute the graded ranks of the derived holonomy algebra, $\mathfrak{H}^{\prime}(\mathcal{A})=\bigoplus_{r \geq 2} \mathfrak{H}_{r}(\mathcal{A})$.

From work of Falk [3], we know that $\operatorname{dim}_{\mathbb{Q}} \mathfrak{H}_{r}(\mathcal{A}) \otimes \mathbb{Q} \geq \sum_{X \in \mathscr{L}_{2}(\mathcal{A})} \phi_{r}\left(F_{\mu(X)}\right)$, for all $r \geq 2$, with equality holding for $r=2$. Guided by these facts, we say that $\mathcal{A}$ is decomposable if the lower bound is attained in degree 3 , for every field $\mathbb{k}$ :

$$
\operatorname{dim}_{\mathbb{k}} \mathfrak{H}_{3}(\mathcal{A}) \otimes \mathbb{k}=\sum_{X \in \mathscr{L}_{2}(\mathcal{A})} \phi_{3}\left(F_{\mu(X)}\right) .
$$


Up to now, an explicit formula for the LCS ranks of an arrangement group has only been known in the case when the intersection lattice is supersolvable [4], or, more generally, hypersolvable [5]. The isomorphism (1.1) leads to a new LCS formula, for the combinatorially defined class of decomposable arrangements:

$$
\prod_{r=1}^{\infty}\left(1-t^{r}\right)^{\phi_{r}(G(\mathcal{A}))}=(1-t)^{|\mathcal{A}|} \prod_{X \in \mathscr{L}_{2}(\mathcal{A})} \frac{1-\mu(X) t}{(1-t)^{\mu(X)}} .
$$

This LCS formula verifies the more general "resonance LCS formula", conjectured in [17], in what is arguably the simplest, yet most basic case.

As a byproduct of our main theorem, we compute in Section 6 the integral Chen Lie algebra of a decomposable arrangement, and we also obtain the Chen analog of decomposition (1.1), thus improving upon results from [2].

1.4. Formula (1.5) is equivalent to $\phi_{r}(G(\mathcal{A}))=\sum_{X \in \mathscr{L}_{2}(\mathcal{A})} \phi_{r}\left(F_{\mu(X)}\right)$, for all $r \geq 2$. In other words, the (higher) LCS ranks behave as if $G(\mathcal{A})$ were to decompose as a direct product of free groups, of ranks dictated by the Möbius function. This happens, for instance, for the class of (hypersolvable, decomposable) arrangements considered in [1], where the arrangement group is always a product of free groups. In general, though, the group of a decomposable arrangement does not decompose in this manner.
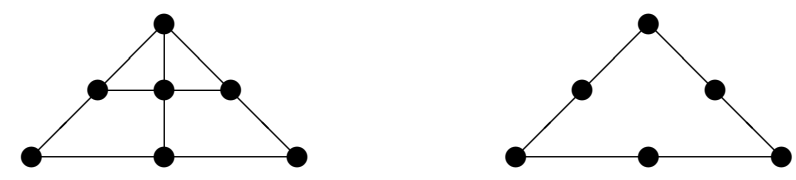

Figure 1. The $X_{2}$ and $X_{3}$ matroids.

For example, consider the $\mathrm{X}_{2}$ and $\mathrm{X}_{3}$ arrangements, whose matroids are depicted in Figure 1. It is readily checked that both arrangements are decomposable (compare with [2], [15]), but not hypersolvable (see Remark 7.3).

For the $\mathrm{X}_{2}$ arrangement, we find that $\phi_{r}(G(\mathcal{A}))=\phi_{r}\left(\left(F_{2}\right)^{\times 5}\right)$, for all $r \geq 2$, yet $\phi_{1}(G(\mathcal{A}))<\phi_{1}\left(\left(F_{2}\right)^{\times 5}\right)$; thus, $G(\mathcal{A}) \neq\left(F_{2}\right)^{\times 5}$.

For the $\mathrm{X}_{3}$ arrangement, we find that $\phi_{r}(G(\mathcal{A}))=\phi_{r}\left(\left(F_{2}\right)^{\times 3}\right)$, for all $r \geq 1$. Even so, $G(\mathcal{A}) \varsubsetneqq\left(F_{2}\right)^{\times 3}$. Indeed, it can be checked that $G(\mathcal{A}) \cong G \times \mathbb{Z}$, where $G$ is the celebrated Stallings group, ${ }^{1}$ equal to the kernel of the projection $\left(F_{2}\right)^{\times 3} \rightarrow \mathbb{Z}$, which sends each standard generator to 1 . As shown in [16], the group $H_{3}(G)$ is

\footnotetext{
${ }^{1}$ As a consequence, we can compute the LCS ranks of the Stallings group: $\phi_{1}(G)=5$, and $\phi_{r}(G)=$ $\phi_{r}\left(\left(F_{2}\right)^{\times 3}\right)$, for $r \geq 2$. In [13], we give an LCS formula that applies to any Bestvina-Brady group associated to a connected flag complex.
} 
not finitely generated. It follows that $G(\mathcal{A})$ does not admit a finite $K(G(\mathcal{A}), 1)$; in particular, $G(\mathcal{A})$ cannot be isomorphic to any finite direct product of free groups of finite rank.

In view of these examples, and of the infinite families of decomposable, nonhypersolvable graphic arrangements from Section 7, we see that the LCS formula (1.5) is a genuinely new formula, with a range of applicability which overlaps only marginally with that of the classical LCS formula.

Acknowledgment. Most of this work was done while the second author visited the Institute of Mathematics of the Romanian Academy in June-July 2003, with partial support from grant CERES/CE4 of the Romanian Ministry of Education and Research.

\section{Decomposable arrangements}

In this section, we delineate the class of decomposable arrangements, and state in detail our main result.

2.1. For an arrangement $\mathcal{A}$, denote by $\mathbb{Z}^{\mathcal{A}}$ the free abelian group on $\mathcal{A}$, with basis $\left\{x_{H} \mid H \in \mathcal{A}\right\}$. For a sub-arrangement $\mathscr{B} \subset \mathcal{A}$, let $\pi_{\mathscr{B}}: \mathbb{Z}^{\mathcal{A}} \rightarrow \mathbb{Z}^{\mathscr{B}}$ be the canonical projection map, defined by $\pi_{\mathscr{B}}\left(x_{H}\right)=x_{H}$, if $H \in \mathscr{B}$, and $\pi_{\mathscr{B}}\left(x_{H}\right)=0$, if $H \notin$ $\mathscr{B}$, and let $\boldsymbol{L}\left(\pi_{\mathscr{B}}\right): \boldsymbol{L}(\mathcal{A}) \rightarrow \boldsymbol{L}(\mathscr{B})$ be its extension to free Lie algebras. Clearly, $\boldsymbol{L}\left(\pi_{\mathscr{B}}\right)(J(\mathcal{A})) \subset J(\mathscr{B})$, and so we get a Lie algebra epimorphism,

$$
\mathfrak{H}\left(\pi_{\mathcal{B}}\right): \mathfrak{H}(\mathcal{A}) \rightarrow \mathfrak{H}(\mathscr{B}) .
$$

For a flat $X \in \mathscr{L}_{2}(\mathcal{A})$, let $\mathcal{A}_{X}=\{H \in \mathcal{A} \mid H \supset X\}$ be the localization of $\mathcal{A}$ at $X$. This is a pencil of $\left|\mathcal{A}_{X}\right|=\mu(X)+1$ hyperplanes. The group $G\left(\mathcal{A}_{X}\right)$ is isomorphic to $F_{\mu(X)} \times \mathbb{Z}$; thus, $\operatorname{gr}\left(G\left(\mathcal{A}_{X}\right)\right) \cong \boldsymbol{L}_{\mu(X)} \times \boldsymbol{L}_{1}$. From the defining relations (1.2), we also have $\mathfrak{H}\left(\mathcal{A}_{X}\right) \cong \boldsymbol{L}_{\mu(X)} \times \boldsymbol{L}_{1}$, and so $\mathfrak{H}\left(\mathcal{A}_{X}\right) \cong \operatorname{gr}\left(G\left(\mathcal{A}_{X}\right)\right)$.

Set $\pi_{X}=\pi_{\mathcal{A}_{X}}$. The maps $\mathfrak{H}\left(\pi_{X}\right): \mathfrak{H}(\mathcal{A}) \rightarrow \mathfrak{H}\left(\mathcal{A}_{X}\right)$ assemble into a Lie algebra map from $\mathfrak{H}(\mathcal{A})$ to the direct product of the holonomy Lie algebras of its localized sub-arrangements:

$$
\pi=\left(\mathfrak{H}\left(\pi_{X}\right)\right)_{X}: \mathfrak{H}(\mathcal{A}) \longrightarrow \prod_{X \in \mathscr{L}_{2}(\mathcal{A})} \mathfrak{H}\left(\mathcal{A}_{X}\right) .
$$

The starting point of our investigation is the following result, to be proved in $\$ 3.2$.

Proposition 2.1. The restriction of $\pi$ to derived subalgebras,

$$
\pi^{\prime}: \mathfrak{H}^{\prime}(\mathcal{A}) \rightarrow \prod_{X \in \mathscr{L}_{2}(\mathcal{A})} \mathfrak{H}^{\prime}\left(\mathcal{A}_{X}\right)
$$

is surjective. 
By comparing ranks of the source and target of $\pi_{r}: \mathfrak{H}_{r}(\mathcal{A}) \rightarrow \prod_{X} \mathfrak{H}_{r}\left(\mathcal{A}_{X}\right)$ for $r \geq 2$, we recover a lower bound for the LCS ranks of an arrangement group, first obtained by M. Falk [3], by other methods.

Corollary 2.2 ([3]). For all $r \geq 2$,

$$
\phi_{r}(G(\mathcal{A})) \geq \sum_{X \in \mathscr{L}_{2}(\mathcal{A})} \phi_{r}\left(F_{\mu(X)}\right) .
$$

2.2. Our main goal here is to understand when the natural map $\pi^{\prime}$ from Proposition 2.1 is, in fact, an isomorphism; in particular, when the inequalities (2.3) become equalities.

It is easy to see that $\pi_{2}$ is always an isomorphism. On the other hand, the maps $\pi_{r}(r \geq 3)$ may not be isomorphisms, as illustrated by the braid arrangements $\mathscr{B}_{\ell}$ in $\mathbb{C}^{\ell}(\ell \geq 4)$. In this case, the LCS formula of Kohno [7] and Falk-Randell [4], when applied to the pure braid group $P_{\ell}=G\left(\mathscr{B}_{\ell}\right)$, shows that inequality (2.3) is strict in degree $r=3$.

This prompts the following definition.

Definition 2.3. Let $r \geq 2$ be an integer, and let $\mathbb{k}$ be a field. We say that $\mathfrak{H}_{r}(\mathscr{A})$ is $\mathbb{k}$-decomposable if

$$
\operatorname{dim}_{\mathbb{k}} \mathfrak{H}_{r}(\mathcal{A}) \otimes \mathbb{k}=\sum_{X \in \mathscr{L}_{2}(\mathcal{A})} \phi_{r}\left(F_{\mu(X)}\right) .
$$

An arrangement $\mathcal{A}$ is decomposable if $\mathfrak{H}_{3}(\mathcal{A})$ is $\mathbb{k}$-decomposable, for every field $\mathbb{k}$.

By Proposition 2.1, $\mathfrak{H}_{r}(\mathscr{A})$ is $\mathbb{k}$-decomposable if and only if $\pi_{r} \otimes \mathbb{k}$ is an isomorphism, whereas $\mathcal{A}$ is decomposable precisely when $\pi_{3}$ is an isomorphism.

2.3. Two other decomposability conditions were considered in [2] and [15]. Let us briefly compare those conditions to ours.

The condition from [2] entails the decomposability of the $I$-adic completion of the Alexander invariant of $G(\mathcal{A})$ as the direct sum of the $I$-adic completions of the Alexander invariants of $G\left(\mathcal{A}_{X}\right)$, taken over $X \in \mathscr{L}_{2}(\mathcal{A})$. It can be shown that this condition on Alexander invariants is equivalent, over $\mathbb{Q}$, to the decomposability of $\mathfrak{H}_{3}(\mathcal{A})$, in the sense of Definition 2.3.

The condition from [15] entails the minimality of the linear strand of the free resolution of the Orlik-Solomon algebra of $\mathcal{A}$ as a module over the corresponding exterior algebra. As stated in [15, Definition 2.10], the MLS condition is equivalent to the $\mathbb{k}$-decomposability of $\mathfrak{H}_{3}(\mathcal{A})$, for $\mathbb{k}$ a field of characteristic 0 . Actually, the only place where the hypothesis char $\mathbb{k}=0$ is needed in that context is to insure 
that $\operatorname{dim}_{\mathbb{k}} \operatorname{gr}_{*}(G(\mathcal{A})) \otimes \mathbb{k}=\operatorname{dim}_{\mathbb{k}} \mathfrak{H}_{*}(\mathcal{A}) \otimes \mathbb{k}$. All the other homological algebra arguments work as well over a field of positive characteristic. Consequently, Theorem 5.6 from [15] gives the following: If $\mathfrak{H}_{3}(\mathcal{A})$ is $\mathbb{k}$-decomposable, then $\mathfrak{H}_{4}(\mathcal{A})$ is $\mathbb{k}$-decomposable. In particular, if $\mathcal{A}$ is decomposable (i.e., $\pi_{3}$ is an isomorphism), then $\pi_{4}$ is an isomorphism.

2.4. Our main result is Theorem 2.4 below, which improves upon the aforementioned result from [15], in several ways. For one, it pushes the range where $\pi_{r}$ is an isomorphism from $r=4$ to infinity. For another, it assembles the graded pieces $\pi_{r}$ $(r \geq 2)$ into a Lie algebra isomorphism between the derived holonomy Lie algebra of $\mathcal{A}$ and a product of derived free Lie algebras. Finally, it gives a new LCS-type formula for the group of a decomposable arrangement, thus verifying Conjecture 5.7 from [15].

Theorem 2.4. Let $\mathcal{A}$ be a decomposable arrangement. Then:

(1) $\operatorname{gr}(G(\mathcal{A})) \cong \mathfrak{H}(\mathcal{A})$, as graded Lie algebras.

(2) $\mathfrak{H}(\mathcal{A})$ is torsion-free, as a graded abelian group.

(3) $\pi^{\prime}: \mathfrak{H}^{\prime}(\mathcal{A}) \longrightarrow \prod_{X \in \mathscr{L}_{2}(\mathcal{A})} \mathfrak{H}^{\prime}\left(\mathcal{A}_{X}\right)$ is an isomorphism of graded Lie algebras.

(4) The LCS ranks $\phi_{r}=\phi_{r}(G(\mathcal{A}))$ are given by the following combinatorial formula:

$$
\prod_{r=1}^{\infty}\left(1-t^{r}\right)^{\phi_{r}}=(1-t)^{b_{1}-b_{2}} \prod_{X \in \mathscr{L}_{2}(\mathcal{A})}(1-\mu(X) t),
$$

where $b_{1}=|\mathcal{A}|$ and $b_{2}=\sum_{X \in \mathscr{L}_{2}(\mathcal{A})} \mu(X)$.

Here is an immediate corollary, already mentioned in the Introduction.

Corollary 2.5. If $\mathcal{A}$ is decomposable, then the associated graded Lie algebra of $G(\mathcal{A})$ decomposes as a direct product of free Lie algebras (in degrees $r \geq 2$ ):

$$
\operatorname{gr}_{\geq 2}(G(\mathcal{A})) \cong \prod_{X \in \mathscr{L}_{2}(\mathcal{A})} \operatorname{gr}_{\geq 2}\left(F_{\mu(X)}\right)
$$

Over the rationals, we can be even more precise: $\mathfrak{H}_{3}(\mathcal{A})$ is decomposable over $\mathbb{Q}$, i.e., $\phi_{3}(G(\mathcal{A}))=\sum_{X \in \mathscr{L}_{2}(\mathcal{A})} \phi_{3}\left(F_{\mu(X)}\right)$, if and only if the derived subalgebra of the rational associated graded Lie algebra of $G(\mathcal{A})$ decomposes as a direct product of derived free Lie algebras over $\mathbb{Q}$ :

$$
(\operatorname{gr}(G(\mathcal{A})) \otimes \mathbb{Q})^{\prime} \cong \prod_{X \in \mathscr{L}_{2}(\mathcal{A})} \boldsymbol{L}_{\mu(X)}^{\prime} \otimes \mathbb{Q} .
$$

This follows from Proposition 4.1 below and formula (1.3). 
Now set $\phi_{r}^{\mathbb{k}}(\mathcal{A}):=\operatorname{dim}_{\mathbb{k}} \mathfrak{H}_{r}(\mathcal{A}) \otimes \mathbb{k}$, for $\mathbb{k}$ a field and $r \geq 1$. The isomorphism (1.3) implies $\phi_{r}^{\mathbb{Q}}(\mathcal{A})=\phi_{r}(G(\mathcal{A}))$, for all $r$. As a consequence of Theorem 2.4, we obtain the following characterization of decomposability, in terms of LCS-type formulas in arbitrary characteristic.

Corollary 2.6. The arrangement $\mathcal{A}$ is decomposable if and only if, for every field $\mathbb{k}$,

$$
\prod_{r=1}^{\infty}\left(1-t^{r}\right)^{\phi_{r}^{\mathbb{k}(\mathcal{A})}}=(1-t)^{b_{1}-b_{2}} \prod_{X \in \mathscr{L}_{2}(\mathcal{A})}(1-\mu(X) t) .
$$

Finally, suppose $\mathcal{A}$ is hypersolvable, with exponents $d_{1}=1, d_{2}, \ldots, d_{\ell}$. The Poincaré polynomial of the quadratic Orlik-Solomon algebra associated to $\mathcal{A}$ is then given by $\bar{P}_{\mathcal{A}}(t)=\prod_{i=1}^{\ell}\left(1+d_{i} t\right)$; see [5, Proposition 3.2]. Putting together the decomposable LCS formula (2.5) and the hypersolvable LCS formula from [5, Theorem C], we obtain the following relationship between the exponents $d_{i}$ and the level-2 Möbius function $\mu: \mathscr{L}_{2}(\mathcal{A}) \rightarrow \mathbb{Z}$ of a decomposable, hypersolvable arrangement $\mathcal{A}$. (We will exploit this relationship in the last section, within the framework of graphic arrangements.)

Corollary 2.7. If $\mathcal{A}$ is both hypersolvable and decomposable, then

$$
\prod_{i=1}^{\ell}\left(1+d_{i} t\right)=(1+t)^{|\mathcal{A}|} \prod_{X \in \mathscr{L}_{2}(\mathcal{A})} \frac{1+\mu(X) t}{(1+t)^{\mu(X)}} .
$$

\section{The $\iota$ map}

In this section, we define the natural candidate for the inverse map to $\pi^{\prime}: \mathfrak{H}^{\prime}(\mathcal{A}) \rightarrow$ $\prod_{X \in \mathscr{L}_{2}(\mathscr{A})} \mathfrak{H}^{\prime}\left(\mathcal{A}_{X}\right)$, and discuss some of its properties.

3.1. Let $\mathcal{B}$ be a sub-arrangement of $\mathcal{A}$. Let $\iota_{\mathbb{B}}: \mathbb{Z}^{\mathscr{B}} \rightarrow \mathbb{Z}^{\mathcal{A}}$ be the canonical inclusion, defined by $\iota_{\mathcal{B}}\left(x_{H}\right)=x_{H}$, and let $\boldsymbol{L}\left(\iota_{\mathscr{B}}\right): \boldsymbol{L}(\mathscr{B}) \rightarrow \boldsymbol{L}(\mathcal{A})$ be its extension to free Lie algebras. In general, the map $L\left(\iota_{\mathscr{B}}\right)$ need not preserve the defining ideals of the holonomy Lie algebras of $\mathcal{B}$ and $\mathcal{A}$.

However, suppose $\mathcal{B}$ is closed in $\mathcal{A}$, i.e., the only linear combinations of defining forms for the hyperplanes in $\mathscr{B}$ which are defining forms for hyperplanes in $\mathcal{A}$ are (up to constants) the defining forms for the hyperplanes in $\mathscr{B}$. Then $\mathscr{L}_{2}(\mathscr{B})=\{X \in$ $\left.\mathscr{L}_{2}(\mathcal{A}) \mid \mathcal{A}_{X} \subset \mathscr{B}\right\}$. Thus, $\boldsymbol{L}\left(\iota_{\mathscr{B}}\right)(J(\mathscr{B})) \subset J(\mathcal{A})$, and so we get a map of graded Lie algebras,

$$
\mathfrak{H}\left(\iota_{\mathcal{B}}\right): \mathfrak{H}(\mathscr{B}) \rightarrow \mathfrak{H}(\mathcal{A}) .
$$

For a flat $X \in \mathscr{L}_{2}(\mathcal{A})$, note that $\mathcal{A}_{X}$ is closed in $\mathcal{A}$. Set $\iota_{X}=\iota_{\mathcal{A}_{X}}$. 
Lemma 3.1. Let $X, Y \in \mathscr{L}_{2}(\mathcal{A})$, and $\mathscr{B} \subset \mathcal{A}$. Then:

(1) $\mathfrak{H}\left(\pi_{X}\right) \circ \mathfrak{H}\left(\iota_{X}\right)=$ id.

(2) $\mathfrak{H}^{\prime}\left(\pi_{\mathcal{B}}\right) \circ \mathfrak{H}^{\prime}\left(\iota_{X}\right)=0$, if $\left|\mathscr{B} \cap \mathcal{A}_{X}\right| \leq 1$.

(3) $\mathfrak{H}^{\prime}\left(\pi_{X}\right) \circ \mathfrak{H}^{\prime}\left(\iota_{Y}\right)=0$, if $X \neq Y$.

Proof. (1) Clearly, $\pi_{X} \circ \iota_{X}$ is the identity map on $\mathbb{Z}^{\mathcal{A}_{X}}$.

(2) For each $r \geq 2$, the group $\mathfrak{H}_{r}\left(\mathcal{A}_{X}\right)$ is generated by elements of the form $x=\left[x_{H_{1}},\left[x_{H_{2}}, \ldots\left[x_{H_{r-1}}, x_{H_{r}}\right] \ldots\right]\right]$, where $H_{1}, \ldots, H_{r}$ are hyperplanes in $\mathcal{A}_{X}$. Now, since $\left|\mathscr{B} \cap \mathcal{A}_{X}\right| \leq 1$, one of those hyperplanes, say $H_{i}$, must not belong to $\mathscr{B}$; otherwise, $H_{1}=H_{2}=\cdots=H_{r}$, and so $x=0$. Hence, by definition, $\pi_{\mathscr{B}}\left(x_{H_{i}}\right)=0$, and so

$$
\mathfrak{H}\left(\pi_{\mathcal{B}}\right) \circ \mathfrak{H}\left(\iota_{X}\right)(x)=\left[\pi_{\mathcal{B}}\left(x_{H_{1}}\right),\left[\pi_{\mathcal{B}}\left(x_{H_{2}}\right), \ldots\left[\pi_{\mathcal{B}}\left(x_{H_{r-1}}\right), \pi_{\mathcal{B}}\left(x_{H_{r}}\right)\right] \ldots\right]\right]=0 .
$$

Thus, $\mathfrak{H}\left(\pi_{\mathcal{B}}\right) \circ \mathfrak{H}\left(\iota_{X}\right)=0$ in degrees $\geq 2$.

(3) If $X \neq Y$, then $\left|\mathcal{A}_{X} \cap \mathcal{A}_{Y}\right| \leq 1$. Hence (2) applies.

3.2. Proof of Proposition 2.1 The maps $\mathfrak{H}\left(\iota_{X}\right)$ define a homomorphism of graded abelian groups,

$$
\iota: \prod_{X \in \mathscr{L}_{2}(\mathcal{A})} \mathfrak{H}\left(\mathcal{A}_{X}\right) \longrightarrow \mathfrak{H}(\mathcal{A})
$$

Let $\iota^{\prime}: \prod_{X} \mathfrak{H}^{\prime}\left(\mathcal{A}_{X}\right) \rightarrow \mathfrak{H}^{\prime}(\mathcal{A})$ be the restriction of $\iota$ to derived subalgebras. The orthogonality relations from Lemma 3.1 imply that $\pi^{\prime} \circ \iota^{\prime}=\mathrm{id}$. Thus, $\pi^{\prime}$ is surjective, and so Proposition 2.1 is proved.

3.3. The following lemma (the proof of which is an exercise in linear algebra) will be used repeatedly later on.

Lemma 3.2. Let $U$ and $\left\{V_{X}\right\}_{X \in X}$ be finite-dimensional vector spaces over a field $\mathbb{k}$, with $|X|$ finite. Set $V=\bigoplus_{X} V_{X}$. Suppose we have linear maps $\pi_{X}: U \rightarrow V_{X}$ and $\iota_{X}: V_{X} \rightarrow U$ such that $\pi_{X} \circ \iota_{Y}=\delta_{X, Y}$. Set $\pi=\left(\pi_{X}\right)_{X}: U \rightarrow V$ and $\iota=\sum_{X} \iota_{X}: V \rightarrow U$. Then, the following conditions are equivalent:

(1) $\pi$ is an isomorphism.

(2) i is surjective.

(3) $\sum_{X} \iota_{X} \circ \pi_{X}=\operatorname{id}_{U}$.

(4) $\operatorname{dim}_{\mathbb{k}} U=\sum_{X} \operatorname{dim}_{\mathbb{k}} V_{X}$. 
3.4. By Lemmas 3.1 and $3.2, \mathfrak{H}_{3}(\mathcal{A})$ is $\mathbb{k}$-decomposable if and only if the map

$$
\iota_{3} \otimes \mathbb{k}: \bigoplus_{X \in \mathscr{L}_{2}(\mathcal{A})} \mathfrak{H}_{3}\left(\mathcal{A}_{X}\right) \otimes \mathbb{k} \longrightarrow \mathfrak{H}_{3}(\mathcal{A}) \otimes \mathbb{k}
$$

is surjective. We use this criterion to show that decomposability is hereditary.

Proposition 3.3. If $\mathscr{B}$ is a sub-arrangement of $\mathcal{A}$, and if $\mathfrak{H}_{3}(\mathcal{A})$ is $\mathbb{k}$-decomposable, then $\mathfrak{H}_{3}(\mathscr{B})$ is also $\mathbb{k}$-decomposable.

Proof. Note that $\mathscr{L}_{2}(\mathscr{B})=\left\{X \in \mathscr{L}_{2}(\mathcal{A})|| \mathcal{A}_{X} \cap \mathscr{B} \mid \geq 2\right\}$. Furthermore, if $X \in$ $\mathscr{L}_{2}(\mathscr{B})$, then $\mathscr{B}_{X}=\mathcal{A}_{X} \cap \mathscr{B}$. Consider the following diagram:

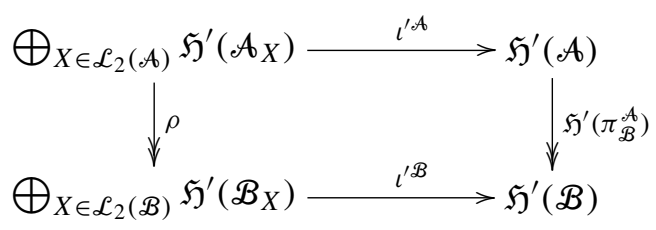

where $\rho$ restricts to $\mathfrak{H}^{\prime}\left(\pi_{\mathcal{B}_{X}}^{\mathcal{A}_{X}}\right): \mathfrak{H}^{\prime}\left(\mathcal{A}_{X}\right) \rightarrow \mathfrak{H}^{\prime}\left(\mathscr{B}_{X}\right)$ if $X \in \mathcal{L}_{2}(\mathscr{B})$, and $\rho=0$ otherwise. Diagram (3.4) commutes. Indeed, if $X \in \mathscr{L}_{2}(\mathscr{B})$, this is clear. If $X \notin$ $\mathscr{L}_{2}(\mathscr{B})$, then $\left|\mathcal{A}_{X} \cap \mathscr{B}\right| \leq 1$, and so, by Lemma $3.1(2), \mathfrak{H}^{\prime}\left(\pi_{\mathscr{B}}^{\mathcal{A}}\right) \circ \mathfrak{H}^{\prime}\left(\iota_{\mathcal{A}_{X}}^{\mathcal{A}}\right)=0$.

Now, if $\mathfrak{H}_{3}(\mathcal{A})$ is $\mathbb{k}$-decomposable, then $\iota_{3}^{\mathcal{A}} \otimes \mathbb{k}$ is surjective. From the commutativity of diagram (3.4), we infer that $\iota_{3}^{\mathcal{B}} \otimes \mathbb{k}$ is also surjective, and we are done.

\section{Surjectivity of $\iota^{\prime}$}

In general, the map $\iota^{\prime}: \prod_{X} \mathfrak{H}^{\prime}\left(\mathcal{A}_{X}\right) \rightarrow \mathfrak{H}^{\prime}(\mathcal{A})$ is not surjective. On the other hand, if $\mathscr{A}$ is decomposable, $\iota^{\prime}$ is surjective. This we show in the next proposition, which is the key to our main result.

Proposition 4.1. Suppose $\mathfrak{H}_{3}(\mathcal{A})$ is $\mathbb{k}$-decomposable. Then

$$
\iota_{r} \otimes \mathbb{k}: \prod_{X} \mathfrak{H}_{r}\left(\mathcal{A}_{X}\right) \otimes \mathbb{k} \longrightarrow \mathfrak{H}_{r}(\mathcal{A}) \otimes \mathbb{k}
$$

is surjective, for all $r \geq 2$.

Proof. For simplicity, we will suppress the field $\mathbb{k}$ from the notation. We will need to establish various commutation relations in $\mathfrak{H}(\mathcal{A})$, between elements in $\mathfrak{H}\left(\iota_{X}\right)\left(\mathfrak{H}\left(\mathcal{A}_{X}\right)\right)$ 
and $\mathfrak{H}\left(\iota_{Y}\right)\left(\mathfrak{H}\left(\mathcal{A}_{Y}\right)\right)$, where $X$ and $Y$ are distinct flats in $\mathscr{L}_{2}(\mathcal{A})$. Again for simplicity, we will suppress the inclusion $\iota$ from the notation, and work in $\mathfrak{H}(\mathcal{A})$.

Since $X \neq Y$, there are two possibilities: either $\mathcal{A}_{X} \cap \mathcal{A}_{Y}=\emptyset$, or $\mathcal{A}_{X} \cap \mathcal{A}_{Y}$ consists of a single hyperplane. Pick $H^{\prime} \in \mathcal{A}_{X}$ and $H^{\prime \prime} \in \mathcal{A}_{Y}$, so that, if $\mathcal{A}_{X}^{*}=$ $\mathcal{A}_{X} \backslash\left\{H^{\prime}\right\}$ and $\mathcal{A}_{Y}^{*}=\mathcal{A}_{Y} \backslash\left\{H^{\prime \prime}\right\}$ are the corresponding deletions, then

$$
\mathcal{A}_{X}^{*} \cap \mathcal{A}_{Y}=\mathcal{A}_{X} \cap \mathcal{A}_{Y}^{*}=\emptyset .
$$

Let us note the following fact, whose proof is immediate, and which will be used repeatedly in the sequel. For any flat $Z \in \mathscr{L}_{2}(\mathcal{A})$, and for any hyperplane $H \in \mathcal{A}_{Z}$,

$$
\mathfrak{H}^{\prime}\left(\mathcal{A}_{Z}\right)=\operatorname{Lie}^{>1}\left(\mathcal{A}_{Z}^{*}\right),
$$

where $\mathcal{A}_{Z}^{*}=\mathcal{A}_{Z} \backslash\{H\}$, and where $\operatorname{Lie}^{r}\left(\mathcal{A}_{Z}^{*}\right)$ denotes the degree $r$ piece of the Lie subalgebra generated by $\left\{x_{K} \mid K \in \mathcal{A}_{Z}^{*}\right\}$ inside $\mathfrak{H}\left(\mathcal{A}_{Z}\right)$.

Here is the first commutation property, for which the decomposability assumption on $\mathfrak{H}_{3}(\mathcal{A})$ is needed in a crucial way.

Claim I. If $H_{i} \in \mathcal{A}_{X}^{*}$ and $c \in \mathfrak{H}_{2}\left(\mathcal{A}_{Y}\right)$, then $\left[x_{H_{i}}, c\right]=0$.

Proof. By (4.2), it is enough to verify the claim for $c \in \operatorname{Lie}^{2}\left(\mathcal{A}_{Y}^{*}\right)$. Apply $\mathfrak{H}\left(\pi_{Z}\right)$, for some $Z \in \mathscr{L}_{2}(\mathcal{A})$. If $Z \neq Y$, we get $\left[\mathfrak{H}\left(\pi_{Z}\right)\left(x_{H_{i}}\right), \mathfrak{H}\left(\pi_{Z}\right)(c)\right]=\left[\mathfrak{H}\left(\pi_{Z}\right)\left(x_{H_{i}}\right), 0\right]=$ 0 . If $Z=Y$, we get $\left[\mathfrak{H}\left(\pi_{Y}\right)\left(x_{H_{i}}\right), c\right]=[0, c]=0$, since $\mathcal{A}_{X}^{*} \cap \mathcal{A}_{Y}=\emptyset$. Thus, $\pi_{3}\left(\left[x_{H_{i}}, c\right]\right)=0$, and so $\left[x_{H_{i}}, c\right]=0$, since, by assumption, $\pi_{3}$ is an isomorphism.

Using Claim I, we obtain the next commutation property.

Claim II. If $b \in \mathfrak{H}_{2}\left(\mathcal{A}_{X}\right)$ and $c \in \mathfrak{H}_{s}\left(\mathcal{A}_{Y}\right)(s \geq 2)$, then $[b, c]=0$.

Proof. As before, we may assume that $b \in \operatorname{Lie}^{2}\left(\mathcal{A}_{X}^{*}\right)$ and $c \in \operatorname{Lie}^{s}\left(\mathcal{A}_{Y}^{*}\right)$. The proof is by induction on $s$. For $s=2$, Claim II follows from Claim I, via the Jacobi identity. For the induction step, take an element $c \in \mathrm{Lie}^{s+1}\left(\mathcal{A}_{Y}^{*}\right)$, and write it as $c=\left[x_{H_{j}}, c^{\prime}\right]$, with $H_{j} \in \mathcal{A}_{Y}^{*}$ and $c^{\prime} \in \operatorname{Lie}^{s}\left(\mathcal{A}_{Y}^{*}\right)$. By the Jacobi identity,

$$
[b, c]=\left[\left[b, x_{H_{j}}\right], c^{\prime}\right]+\left[x_{H_{j}},\left[b, c^{\prime}\right]\right] .
$$

Note that $\left[b, x_{H_{j}}\right]=0$ by Claim I, and $\left[b, c^{\prime}\right]=0$ by induction. Thus, $[b, c]=0$.

Finally, using both Claims I and II, we prove the following key commutation property.

Claim III. If $H_{i} \in \mathcal{A}_{X}^{*}$ and $c \in \mathfrak{H}_{S}\left(\mathcal{A}_{Y}\right)(s \geq 2)$, then $\left[x_{H_{i}}, c\right] \in \mathfrak{H}_{s+1}\left(\mathcal{A}_{Y}\right)$. 
Proof. The proof is by induction on $s$. The case $s=2$ follows from Claim I. For the induction step, take an element $c=\left[x_{H_{j}}, c^{\prime}\right] \in \mathfrak{H}_{s+1}\left(\mathcal{A}_{Y}\right)$, with $H_{j} \in \mathcal{A}_{Y}$ and $c^{\prime} \in \mathfrak{H}_{S}\left(\mathcal{A}_{Y}\right)$. By the Jacobi identity,

$$
\left[x_{H_{i}}, c\right]=\left[\left[x_{H_{i}}, x_{H_{j}}\right], c^{\prime}\right]+\left[x_{H_{j}},\left[x_{H_{i}}, c^{\prime}\right]\right] .
$$

By induction, $\left[x_{H_{i}}, c^{\prime}\right] \in \mathfrak{H}_{s+1}\left(\mathcal{A}_{Y}\right)$, and so $\left[x_{H_{j}},\left[x_{H_{i}}, c^{\prime}\right]\right] \in \mathfrak{H}_{s+2}\left(\mathcal{A}_{Y}\right)$.

On the other hand, since $H_{i} \neq H_{j}$, there exists a flat $Z \in \mathscr{L}_{2}(\mathcal{A})$ such that $\left\{H_{i}, H_{j}\right\} \subset \mathcal{A}_{Z}$, and so $\left[x_{H_{i}}, x_{H_{j}}\right] \in \mathfrak{H}_{2}\left(\mathcal{A}_{Z}\right)$. If $Z=Y$, then $\left[\left[x_{H_{i}}, x_{H_{j}}\right], c^{\prime}\right] \in$ $\left[\mathfrak{H}_{2}\left(\mathscr{A}_{Y}\right), \mathfrak{H}_{s}\left(\mathscr{A}_{Y}\right)\right] \subset \mathfrak{H}_{s+2}\left(\mathscr{A}_{Y}\right)$. If $Z \neq Y$, then $\left[\left[x_{H_{i}}, x_{H_{j}}\right], c^{\prime}\right]=0$, by Claim II. Either way, we conclude that $\left[x_{H_{i}}, c\right] \in \mathfrak{H}_{s+2}\left(\mathcal{A}_{Y}\right)$.

Having established the above claims, we are now ready to prove Proposition 4.1, by induction on $r$. For $r=2$, the map $\iota_{2}$ is surjective, since $\pi_{2} \circ \iota_{2}=\mathrm{id}$, and $\pi_{2}$ is an isomorphism (for arbitrary $\mathcal{A}$ ). For the induction step, it is plainly enough to show that

$$
\left[x_{H}, c\right] \in \mathfrak{H}_{r+1}\left(\mathcal{A}_{Y}\right),
$$

for any $H \in \mathcal{A}$ and $c \in \mathfrak{H}_{r}\left(\mathcal{A}_{Y}\right)$, where $Y \in \mathscr{L}_{2}(\mathcal{A})$ and $r \geq 2$.

If $H \in \mathcal{A}_{Y}$, this is clear. Assuming $H \notin \mathcal{A}_{Y}$, pick any $H^{\prime \prime} \in \mathcal{A}_{Y}$, and set $X=H \cap H^{\prime \prime} \in \mathscr{L}_{2}(\mathcal{A})$. Note that $X \neq Y$, and $\mathcal{A}_{X}^{*}=\mathcal{A}_{X} \backslash\left\{H^{\prime \prime}\right\}$. Hence, $H \in \mathcal{A}_{X}^{*}$, and (4.3) now follows from Claim III. The proof of Proposition 4.1 is thus complete.

\section{Proof of Theorem 2.4}

We are now in the position to prove our main result.

Let $\mathcal{A}$ be an arbitrary arrangement. Recall we defined in $\$ 2.1$ a homomorphism of graded Lie algebras, $\pi: \mathfrak{H}(\mathscr{A}) \rightarrow \prod_{X \in \mathscr{L}_{2}(\mathcal{A})} \mathfrak{H}\left(\mathcal{A}_{X}\right)$. Recall also we defined in $\$ 3.2$ a homomorphism of graded abelian groups, $\iota: \prod_{X \in \mathscr{L}_{2}(\mathcal{A})} \mathfrak{H}\left(\mathcal{A}_{X}\right) \rightarrow \mathfrak{H}(\mathcal{A})$, with the property that $\pi^{\prime} \circ \iota^{\prime}=\mathrm{id}$, which showed that $\pi^{\prime}$ is an epimorphism.

Now suppose $\mathcal{A}$ is decomposable. By Proposition 4.1, each map $\iota_{r}(r \geq 2)$ is surjective. By Lemmas 3.1 and 3.2, each map $\pi_{r}(r \geq 2)$ is an isomorphism. Hence, $\pi^{\prime}$ is an isomorphism of Lie algebras, with inverse $\iota^{\prime}$. This proves Part (3) of Theorem 2.4 .

Part (2) follows at once from (3), and the fact that each Lie algebra $\mathfrak{H}\left(\mathcal{A}_{X}\right) \cong$ $\boldsymbol{L}_{\mu(X)} \times \boldsymbol{L}_{1}$ is torsion-free.

Part (1) follows from (2), together with (1.3) and [9, Proposition 5.1].

Part (4) follows from (3), together with (1.3) and the discussion from [15, §1.5]. 


\section{Decomposable Chen Lie algebras}

Another, much coarser approximation to the associated graded Lie algebra of a group is its Chen Lie algebra. We now study the effect of the decomposability condition on the Chen Lie algebra of an arrangement group.

Given a finitely-generated group $G$, let $G / G^{\prime \prime}$ be the quotient by its second derived subgroup. We call the associated graded Lie algebra $\operatorname{gr}\left(G / G^{\prime \prime}\right)$, the Chen Lie algebra of $G$. Set $\theta_{k}(G)=\operatorname{rankgr}_{k}\left(G / G^{\prime \prime}\right)$. Plainly, $\theta_{k}(G)=\phi_{k}(G)$ for $k \leq 3$, and $\theta_{k}(G) \leq \phi_{k}(G)$ for $k>3$.

Now suppose $G(\mathcal{A})$ is an arrangement group. Then, as shown in [12, Theorem 11.1], there is an isomorphism of graded Lie algebras,

$$
\operatorname{gr}\left(G(\mathcal{A}) / G^{\prime \prime}(\mathcal{A})\right) \otimes \mathbb{Q} \cong\left(\mathfrak{H}(\mathcal{A}) / \mathfrak{H}^{\prime \prime}(\mathcal{A})\right) \otimes \mathbb{Q} .
$$

Let $B(\mathcal{A})=\mathfrak{H}^{\prime}(\mathcal{A}) / \mathfrak{H}^{\prime \prime}(\mathcal{A})$ be the infinitesimal Alexander invariant of $\mathcal{A}$. Taking graded ranks on both sides of (6.1), we find

$$
\theta_{k}(G(\mathcal{A}))=\operatorname{rank} B_{k}(\mathcal{A}) \quad \text { for all } k \geq 2
$$

Recall once more the surjective map of graded Lie algebras from Proposition 2.1, $\pi^{\prime}: \mathfrak{H}^{\prime}(\mathcal{A}) \rightarrow \prod_{X \in \mathscr{L}_{2}(\mathcal{A})} \mathfrak{H}^{\prime}\left(\mathscr{A}_{X}\right)$. By abelianization, we obtain an epimorphism of graded abelian groups,

$$
B(\pi): B(\mathcal{A}) \longrightarrow \bigoplus_{X \in \mathscr{L}_{2}(\mathcal{A})} B\left(\mathcal{A}_{X}\right)
$$

By comparing graded ranks of the source and target of $B(\pi)$, we recover a lower bound for the Chen ranks of an arrangement group, first obtained in [2] by other methods.

Corollary 6.1 ([2]). For all $r \geq 2$,

$$
\theta_{r}(G(\mathcal{A})) \geq \sum_{X \in \mathcal{L}_{2}(\mathcal{A})} \theta_{r}\left(F_{\mu(X)}\right)
$$

where $\theta_{r}\left(F_{n}\right)=(r-1)\left(\begin{array}{c}n+r-2 \\ r\end{array}\right)$.

Note that $B_{2}(\pi)=\pi_{2}$ is an isomorphism, and thus equality holds in (6.4) for $r=2$. For $r \geq 3$, though, the inequality can well be strict; see again [2].

As another application of our methods, we provide a complete description of the Chen Lie algebra of a decomposable arrangement. 
Theorem 6.2. If $\mathcal{A}$ is decomposable, then:

(1) $\operatorname{gr}\left(G(\mathcal{A}) / G^{\prime \prime}(\mathcal{A})\right)=\mathfrak{H}(\mathcal{A}) / \mathfrak{H}^{\prime \prime}(\mathcal{A})$, as graded Lie algebras over $\mathbb{Z}$.

(2) $\operatorname{gr}\left(G(\mathcal{A}) / G^{\prime \prime}(\mathcal{A})\right)$ is torsion-free, as a graded abelian group.

(3) The Chen ranks of $G(\mathcal{A})$, for $r \geq 2$, are given by

$$
\theta_{r}(G(\mathcal{A}))=\sum_{X \in \mathscr{L}_{2}(\mathcal{A})} \theta_{r}\left(F_{\mu(X)}\right)
$$

Proof. For any flat $X \in \mathscr{L}_{2}(\mathcal{A})$, we have $B\left(\mathcal{A}_{X}\right)=\boldsymbol{L}_{\mu(X)}^{\prime} / \boldsymbol{L}_{\mu(X)}^{\prime \prime}$, which is known to be torsion-free. Now, since $\mathcal{A}$ is decomposable, Theorem 2.4(3) implies that $B(\pi)$ is an isomorphism, and consequently $B(\mathcal{A})$ is torsion-free, as well. Hence, $\mathfrak{H}(\mathcal{A}) / \mathfrak{H}^{\prime \prime}(\mathcal{A})$ is also torsion-free. Parts (1) and (2) now follow from Theorem B in [12]. Part (3) follows from the fact that $B(\pi)$ is an isomorphism, and (6.2).

Formula (6.5) was derived by other methods in [2], under the decomposability condition from that paper.

\section{Decomposable graphic arrangements}

To a (simple) graph $\mathrm{G}$, with vertex set $\mathrm{V}=\{1, \ldots, \ell\}$ and edge set $\mathrm{E}$, there corresponds a graphic arrangement in $\mathbb{C}^{\ell}$, denoted by $\mathcal{A}_{\mathrm{G}}$. The hyperplane corresponding to an edge $e=(i, j)$ is $H_{e}=\left\{z_{i}-z_{j}=0\right\}$. For example, if $\mathrm{G}=K_{\ell}$, the complete graph on $\ell$ vertices, then $\mathcal{A}_{K_{\ell}}=\mathscr{B}_{\ell}$, the braid arrangement in $\mathbb{C}^{\ell}$.

For each flat $X \in \mathscr{L}_{2}\left(\mathcal{A}_{G}\right)$, there are either 2 or 3 hyperplanes containing $X$. Under the identification $\mathcal{A}_{\mathrm{G}}=\mathrm{E}$, a flat of size 3 of corresponds to a triangle in the graph, while a flat of size 2 corresponds to a pair of edges which is not included in any element of the triangle-set $\mathrm{T}$. Thus, the holonomy Lie algebra of $\mathcal{A}_{\mathrm{G}}$ can be identified with the quotient of the free Lie algebra on variables $e \in \mathrm{E}$ by the corresponding ideal of quadratic relations:

$$
\mathfrak{H}(\mathrm{G})=\boldsymbol{L}(\mathrm{E}) / \text { ideal }\left\{\begin{array}{ll}
{\left[e_{1}, e_{2}+e_{3}\right],} & \text { if }\left\{e_{1}, e_{2}, e_{3}\right\} \in \mathrm{T} \\
{\left[e_{1}, e_{2}\right],} & \text { if }\left\{e_{1}, e_{2}, e\right\} \notin \mathrm{T} \text { for all } e \in \mathrm{E}
\end{array}\right\} .
$$

As shown in [15], the $\mathbb{Q}$-decomposability condition for a graphic arrangement can be read off the graph itself, as the absence of complete quadrangles in G. We present a strengthened form of this result, which nicely illustrates our methods.

Proposition 7.1. For a graphic arrangement $\mathcal{A}_{\mathrm{G}}$, the following conditions are equivalent:

(1) $\mathcal{A}_{\mathrm{G}}$ is decomposable. 
(2) $\mathfrak{H}_{3}(\mathrm{G})$ is decomposable over some field $\mathbb{k}$.

(3) G contains no complete subgraphs on 4 vertices.

Proof. The implication (1) $\Rightarrow(2)$ is obvious.

To show $(2) \Rightarrow(3)$, suppose $K_{4}$ is a subgraph of $\mathrm{G}$. Then, the braid arrangement $\mathscr{B}=\mathcal{A}_{K_{4}}$ is a sub-arrangement of $\mathcal{A}_{\mathrm{G}}$. But $\mathcal{B}$ is not $\mathbb{k}$-decomposable, for any field $\mathbb{k}$. Indeed, $\sum_{X \in \mathscr{L}_{2}(\mathscr{B})} \phi_{3}\left(F_{\mu(X)}\right)=8$, whereas $\operatorname{dim}_{\mathbb{k}} \mathfrak{H}_{3}(\mathscr{B}) \otimes \mathbb{k}=10$ (see [7] for the case $\mathbb{k}=\mathbb{Q}$, and [5] for the general case). This contradicts Proposition 3.3.

To show $(3) \Rightarrow(1)$, we must check that $\mathfrak{H}_{3}(\mathrm{G})$ is spanned by $\left\{\iota_{\tau}\left(\mathfrak{H}_{3}(\tau)\right) \mid \tau \in \mathrm{T}\right\}$, where $\iota_{\tau}: \mathfrak{H}(\tau) \rightarrow \mathfrak{H}(\mathrm{G})$ is the natural inclusion. As an abelian group, $\mathfrak{H}_{3}(\mathrm{G})$ is generated by elements of the form $x=\left[e_{1},\left[e_{2}, e_{3}\right]\right]$. Note that the edges $e_{2}, e_{3}$ must belong to a common triangle, say, $\tau$, for, otherwise, $\left[e_{2}, e_{3}\right]=0$ in $\mathfrak{H}(\mathrm{G})$. If $e_{1} \in \tau$, then clearly $x \in \iota_{\tau}\left(\mathfrak{H}_{3}(\tau)\right)$. If $e_{1} \notin \tau$, we will show that $x=0$, and that will finish the proof.

First, we claim that there are two edges, $e$ and $e^{\prime}$, in $\tau$ such that

$$
\left[e_{1}, e\right]=\left[e_{1}, e^{\prime}\right]=0 .
$$

To verify the claim, denote by $\mathrm{G}_{0}$ the subgraph supported on the vertices of $\tau$ and $e_{1}$. Since $e_{1} \notin \tau$, there are two possibilities:

(a) $\mathrm{G}_{0}$ has 5 vertices. Then $\left[e_{1}, e\right]=0$, for any edge $e \in \tau$.

(b) $\mathrm{G}_{0}$ has 4 vertices. Since by assumption $\mathrm{G}_{0} \neq K_{4}$, again there are two possibilities:

(b $\left.b_{1}\right) \mathrm{G}_{0}$ has 4 edges. Then $\left[e_{1}, e\right]=0$, for any edge $e \in \tau$.

$\left(b_{2}\right) G_{0}$ has 5 edges. Then $\mathrm{G}_{0}$ is the union of two triangles, with an edge in common. If $e, e^{\prime}$ are the other two edges in $\tau$, then $\left[e_{1}, e\right]=\left[e_{1}, e^{\prime}\right]=0$.

Thus, (7.2) holds in all cases.

Now, applying (4.2) to $\tau^{*}=\left\{e, e^{\prime}\right\}$, we see that $\left[e_{2}, e_{3}\right]$ is a multiple of $\left[e, e^{\prime}\right]$ in $\mathfrak{H}_{2}(\tau)$. Hence, by (7.2) and the Jacobi identity, $x=0$.

Let $\kappa_{s}=\kappa_{s}(\mathrm{G})$ be the number of $K_{s+1}$ subgraphs of G; for example, $\kappa_{0}=|\mathrm{V}|$, $\kappa_{1}=|\mathrm{E}|, \kappa_{2}=|\mathrm{T}|$. If $\kappa_{3}=0$, then, by Proposition 7.1 and Theorem 2.4, we have

$$
\prod_{r=1}^{\infty}\left(1-t^{r}\right)^{\phi_{r}}=(1-t)^{\kappa_{1}-2 \kappa_{2}}(1-2 t)^{\kappa_{2}} .
$$

We now provide concrete examples where this LCS formula gives new information. For that, we need graphs which are not chordal (i.e., supersolvable), or, more generally, hypersolvable (in the sense of [11]), since otherwise, previously known formulas apply. 
Proposition 7.2. Let $\mathrm{G}$ be a graph with $\kappa_{1} \leq 2 \kappa_{2}$ and $\kappa_{3}=0$. Then $\mathcal{A}_{\mathrm{G}}$ is decomposable, but not hypersolvable.

Proof. Since $\kappa_{3}=0$, the arrangement $\mathcal{A}_{\mathrm{G}}$ is decomposable. If $\mathcal{A}_{\mathrm{G}}$ were hypersolvable, then, by the LCS formula from [5], $\prod_{r=1}^{\infty}\left(1-t^{r}\right)^{\phi_{r}}=(1-t) P(t)$, for some polynomial $P$. In view of (7.3), this can only happen when $\kappa_{1}-2 \kappa_{2}>0$.

Remark 7.3. Let $\mathcal{A}$ be an arrangement (not necessarily graphic) for which the Möbius function takes only the values 1 and 2 on $\mathscr{L}_{2}(\mathcal{A})$. Set

$$
\kappa_{1}=|\mathcal{A}| \quad \text { and } \quad \kappa_{2}=\left|\left\{X \in \mathscr{L}_{2}(\mathcal{A}) \mid \mu(X)=2\right\}\right| .
$$

The same argument as in Proposition 7.2 shows the following: If $\mathcal{A}$ is decomposable and hypersolvable, then $\kappa_{1}-2 \kappa_{2}>0$.
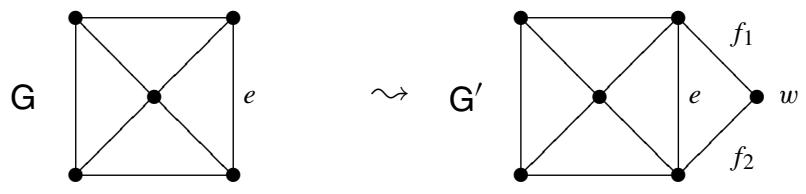

Figure 2. Coning an edge.

Now suppose $\mathrm{G}$ is a graph with $\kappa_{1}-2 \kappa_{2} \leq 0$ and $\kappa_{3}=0$. One can create a new graph, $\mathrm{G}^{\prime}$, with the same properties, as follows. Choose an edge $e$ of G, pick a new vertex $w$, and join it by edges $f_{1}$ and $f_{2}$ to the endpoints of $e$, as in Figure 2. Clearly, $\mathrm{V}^{\prime}=\mathrm{V} \cup\{w\}, \mathrm{E}^{\prime}=\mathrm{E} \cup\left\{f_{1}, f_{2}\right\}, \mathrm{T}^{\prime}=\mathrm{T} \cup\left\{e, f_{1}, f_{2}\right\}$, and there are no complete quadrangles introduced. Thus, $\kappa_{1}^{\prime}-2 \kappa_{2}^{\prime}=\left(\kappa_{1}+2\right)-2\left(\kappa_{2}+1\right) \leq 0$, and $\kappa_{3}^{\prime}=0$. Moreover, it is easy to check that $\mathcal{A}_{\mathrm{G}}$ is solvable in $\mathcal{A}_{\mathrm{G}^{\prime}}$, in the sense of [5].

This permits us to create infinite families of graphs satisfying the hypothesis of Proposition 7.2. For instance, start with the graph $\mathrm{G}^{0}=\mathrm{G}$ from the above figure (see also [15, Example 6.14]), and define inductively a sequence of graphs $\left\{\mathrm{G}^{i}\right\}$ by $\mathrm{G}^{i}=$ $\left(\mathrm{G}^{i-1}\right)^{\prime}$. Since $\mathrm{G}$ satisfies $\kappa_{1}-2 \kappa_{2}=\kappa_{3}=0$, all the graphic arrangements $\mathcal{A}_{\mathrm{G}^{i}}$ are decomposable, but not hypersolvable. By (7.3), the LCS ranks of the corresponding arrangement groups are given by $\prod_{r=1}^{\infty}\left(1-t^{r}\right)^{\phi_{r}}=(1-2 t)^{i+4}$.

Remark 7.4. To the best of our knowledge, the decomposable arrangements discussed in this paper provide the first non-hypersolvable examples where the LCS ranks $\phi_{r}$ are computed for all values of $r$. Note that the two graphic arrangements in Examples 3.7 and 5.4 from [14] are hypersolvable. Indeed, the two underlying graphs can be obtained from hypersolvable graphs, by iterating the above construction: the first one, starting from a 4-cycle, and the second one, starting from the $K_{4}$ graph. As 
such, both arrangements are hypersolvable, cf. $[11, \S 6]$. The first one has rank 4 and exponents $\{1,1,1,1,2\}$; the second one has rank 6 and exponents $\{1,2,2,2,2,3\}$, and thus is actually supersolvable (by [5, Theorem D]), despite a claim to the contrary in [14].

Note added in proof. Using the holonomy Lie algebra approach, P. Lima-Filho and H. Schenck have recently announced in [8] a proof of the LCS formula for graphic arrangements, as conjectured in [15].

\section{References}

[1] A. D. R. Choudary, A. Dimca, S. Papadima, Some analogs of Zariski's theorem on nodal line arrangements. Algebr. Geom. Topol. 5 (2005), 691-711. Zbl 1081.32018 MR 2153112

[2] D. Cohen, A. Suciu, Alexander invariants of complex hyperplane arrangements. Trans. Amer. Math. Soc. 351 (1999), 4043-4067. Zbl 0945.20024 MR 1475679

[3] M. Falk, The cohomology and fundamental group of a hyperplane complement. In Singularities (Iowa City, IA, 1986), Contemp. Math. 90, Amer. Math. Soc, Providence, RI, 1989, 55-72. Zbl 0697.55013 MR 1000594

[4] M. Falk, R. Randell, The lower central series of a fiber-type arrangement. Invent. Math. 82 (1985), 77-88. Zbl 0574.55010 MR 0808110

[5] M. Jambu, S. Papadima, A generalization of fiber-type arrangements and a new deformation method. Topology 37 (1998), 1135-1164. Zbl 0988.52031 MR 1632975

[6] T. Kohno, On the holonomy Lie algebra and the nilpotent completion of the fundamental group of the complement of hypersurfaces. Nagoya Math. J. 92 (1983), 21-37. Zbl 0503.57001 MR 0726138

[7] —, Série de Poincaré-Koszul associée aux groupes de tresses pures. Invent. Math. 82 (1985), 57-75. Zbl 0574.55009 MR 0808109

[8] P. Lima-Filho, H. Schenck, Holonomy Lie algebras and the LCS formula for graphic arrangements. Preprint, 2005.

[9] M. Markl, S. Papadima, Homotopy Lie algebras and fundamental groups via deformation theory. Ann. Inst. Fourier 42 (1992), 905-935. Zbl 0760.55010 MR 1196099

[10] J. Morgan, The algebraic topology of smooth algebraic varieties. Inst. Hautes Études Sci. Publ. Math. 48 (1978), 137-204. Zbl 0401.14003 MR 0516917

[11] S. Papadima, A. Suciu, Higher homotopy groups of complements of hyperplane arrangements. Adv. Math. 165 (2002), 71-100. Zbl 1019.52016 MR 1880322

[12] -, Chen Lie algebras. Internat. Math. Research Notices 2004 (2004), 1057-1086. Zbl 1076.17007 MR 2037049

[13] —, Algebraic invariants for Bestvina-Brady groups. Preprint, 2006; arXiv:math.GR/ 0603240.

[14] I. Peeva, Hyperplane arrangements and linear strands in resolutions. Trans. Amer. Math. Soc. 355 (2002), 609-618. Zbl 01821253 MR 1932716 
[15] H. Schenck, A. Suciu, Lower central series and free resolutions of hyperplane arrangements. Trans. Amer. Math. Soc. 354 (2002), 3409-3433. Zbl 1057.52015 MR 1911506

[16] J. Stallings, A finitely presented group whose 3-dimensional integral homology is not finitely generated. Amer. J. Math. 85 (1963), 541-543. Zbl 0122.27301 MR 0158917

[17] A. Suciu, Fundamental groups of line arrangements: Enumerative aspects. In Advances in algebraic geometry motivated by physics, Contemp. Math. 276, Amer. Math. Soc, Providence, RI, 2001, 43-79. Zbl 0998.14012 MR 1837109

[18] D. Sullivan, Infinitesimal computations in topology. Inst. Hautes Études Sci. Publ. Math. 47 (1977), 269-331. Zbl 0374.57002 MR 0646078

Received January 2, 2005

Stefan Papadima, Institute of Mathematics of the Academy, P.O. Box 1-764,

014700 Bucharest, Romania

E-mail: Stefan.Papadima@imar.ro

Alexander I. Suciu, Department of Mathematics, Northeastern University, Boston, MA 02115, U.S.A.

E-mail: a.suciu@neu.edu

URL: http://www.math.neu.edu/ suciu 